\title{
PRESENCIA DE Campylobacter spp EN CANALES Y CIEGOS DE POLLOS DE ENGORDE EN LIMA, PERÚ
}

\author{
Presence of Campylobacter spp in Poultry meat and Cecum in Lima, Peru
}

Juan Lucas L. ${ }^{1,2}$, Miguel Vilca L. ${ }^{1}$, Daphne Ramos D. ${ }^{1}$

\section{Resumen}

El objetivo del trabajo fue determinar la presencia de Campylobacter spp en canales y ciegos de pollos de engorde comercializados en Lima Metropolitana a través de tres centros de beneficio clandestino. Se muestrearon 90 aves, obteniendo 30 muestras de ciego y 60 muestras de enjuagues de canales. Las muestras fueron sembradas directamente en medio selectivo mCCDA, a $42{ }^{\circ} \mathrm{C}$ por $48 \mathrm{~h}$ en atmósfera microaerofílica y se usaron pruebas bioquímicas para confirmar las especies termotolerantes. El 16.7\% de las canales fueron positivas a Campylobacter spp, donde el $60 \%$ fue con C. jejuni y $40 \%$ con $C$. coli. Además, el $26.7 \%$ de muestras de ciego fueron positivas, siendo el $72.7 \%$ a $C$. jejuni y $27.2 \%$ a C. coli.

Palabras clave: Campylobacter jejuni, Campylobacter coli, centros de beneficio clandestino, superficie de canales, ciego

\section{Abstract}

The aim of this study was to determine the presence of Campylobacter spp in meat and cecum of broilers sold in three illegal abattoirs in Lima, Peru. For this purpose, 90 broilers were selected, collecting 60 samples of body carcasses by the rinse method and 30 samples of cecum. The samples were cultured in mCCDA agar at $42{ }^{\circ} \mathrm{C}$ for $48 \mathrm{~h}$ under microaerophilic atmosphere, and biochemical tests were used to identify thermotolerant Campylobacter species. The results showed that $16.7 \%$ of carcasses were positive, where $60 \%$ were $C$. jejuni and $40 \%$ C. coli. In cecum samples, $26.7 \%$ were positive to Campylobacter spp, where $72.7 \%$ were $C$. jejuni and $27.2 \%$ C. coli.

Key words: Campylobacter jejuni, Campylobacter coli, illegal abattoirs, surface carcasses, caeca

${ }^{1}$ Laboratorio de Salud Pública y Salud Ambiental, Facultad de Medicina Veterinaria, Universidad Nacional Mayor de San Marcos, Lima

${ }^{2}$ E-mail:jrlucas.pe@gmail.com

Recibido: 9 de abril de 2012

Aceptado para publicación: 17 de febrero de 2013 


\section{INTRODUCCIÓN}

La campylobacteriosis es una enfermedad zoonótica causante de gastroenteritis humana, cuya incidencia reporta las mayores tasas entre las enfermedades transmitidas por alimentos (ETA). El agente causal es una bacteria termotolerante del género Campylobacter. Más del $90 \%$ de las infecciones por este género corresponden a Campylobacter jejuni y entre 5 a $10 \%$ a Campylobacter coli (Adedayo y Kirkpatrick, 2008). La dosis infectiva en el humano es baja (Robinson, 1981) y las secuelas de la infección son potencialmente serias. En el Perú, el $C$. jejuni tiene un importante rol como agente etiológico causante de diarrea aguda acuosa en niños (Murga et al. 1993; Perales et al., 2002; Oberhelman et al., 2003).

El Campylobacter está presente en el tracto gastrointestinal de los animales de sangre caliente. Se encuentra como comensal sin manifestar síntomas en el hospedador y es excretado de manera continua en heces. Sus principales reservorios lo constituyen los animales de abasto, roedores, animales de compañía, productos marinos, agua contaminada y productos vegetales en contacto con la materia fecal (Park et al., 1991).

Estudios epidemiológicos y moleculares han demostrado que la mayoría de los casos de infecciones humanas por Campilobacter, los de tipo esporádico, tienen como principal factor de riesgo a la carne de pollo, ya sea por su consumo insuficientemente cocido o por la contaminación cruzada de otros alimentos, o por el contacto directo o indirecto con esta carne cruda durante el manejo, preparación y consumo (Friedman et al., 2004; Humphrey et al., 2007; Lindmark et al., 2009; Suzuki y Yamamoto, 2009; EFSA, 2010).

La enfermedad también se encuentra asociada al enorme consumo de esta ave. En Bélgica, durante la prohibición de la comercialización de carne de pollo en 1999 , hubo una reducción del $40 \%$ de los casos de campylobacteriosis humana, incidencia que se elevó una vez levantada la prohibición (Vellinga y Van Loock, 2002). En el Perú, y en especial en Lima, la población tiene una demanda cada vez mayor de carne de pollo, siendo actualmente la principal fuente proteica (27.6 kg de carne por habitante al año), lo que se refleja en el crecimiento de la producción avícola (MINAG, 2010).

El Campylobacter spp como causa de enteritis humana está bien establecido; sin embargo, en los países en desarrollo no están bien esclarecidas las vías de transmisión (WHO, 2000). Por ello, las investigaciones recientes están dirigidas a entender la forma de transmisión del Campylobacter en la cadena de alimentos para consumo humano. En el Perú se dispone de estudios que señalan la presencia de $C$. jejuni como causante de enfermedades gastroentéricas en la población susceptible (Murga et al., 1993; Perales et al., 2002; Oberhelman et al., 2003), aunque aún no se ha dimensionado el real problema en la Salud Pública en la región (Fernández, 2011). Para poder evaluar el riesgo de contraer una infección a través de la carne de pollo, se precisa primeramente determinar la presencia del patógeno. El presente trabajo identifica los Campylobacter termófilos presentes en el ciego y canales de los pollos de carne en los principales centros de acopio de Lima que fungen de centros de beneficio no autorizados en Lima, Perú.

\section{Materiales y Métodos}

\section{Lugar de Estudio y Muestras}

Se colectaron al azar muestras de canales y ciegos de pollos beneficiados en tres centros de acopio de Lima Metropolitana (ubicados en los distritos de cercado de Lima, San Luis y San Martín de Porres), durante junio a setiembre de 2009. Según la normativa vigente, estos centros deben cumplir las características sanitarias adecuadas para la distribución y comercialización de las aves 
vivas; sin embargo, estos centros fungen, además, como centros de beneficio sin contar con estándares higiénico-sanitarios adecuados. Solo una pequeña proporción de los pollos que se consumen en Lima son beneficiados en centros autorizados (Málaga, 2011), y fue por ello que las muestras fueron colectadas en estos grandes centros de acopio que benefician las aves en forma clandestina.

El tamaño de muestra se obtuvo utilizando la fórmula para poblaciones infinitas (Daniel, 1996) con un nivel de confianza del $90 \%$, usando $82.3 \%(\mathrm{~d}=0.1)$ y $37 \%(\mathrm{~d}=0.15)$ como prevalencia de referencia para canales (Suzuki y Yamamoto, 2009) y heces (Tresierra-Ayala et al., 1996) de pollos, resultando en un mínimo de 40 y 29 muestras, respectivamente. Semanalmente, por un periodo de cinco semanas, y siempre al inicio de la faena, se tomaron tres muestras de cada centro de acopio (un ciego y dos canales), procurando tomar aves de diferentes lotes.

Las canales se guardaron en bolsas plásticas donde se les remojó y masajeó manualmente por 2 minutos con $150 \mathrm{ml}$ de agua peptonada al $0.1 \%$ (método del enjuague), y esta solución de enjuague fue vertida en envases estériles. Asimismo, los ciegos fueron cortados con tijeras, inmediatamente después del proceso de eviscerado, y colocados en una bolsa plástica. Los envases y las bolsas con los ciegos fueron transportados al laboratorio en una caja térmica con hielo $\left(4-8^{\circ} \mathrm{C}\right)$.

\section{Aislamiento e Identificación Bioquímica}

Las muestras fueron procesadas en el Laboratorio de de Salud Pública y Salud Ambiental, Facultad de Medicina Veterinaria, Universidad Nacional Mayor de San Marcos, Lima. Se extrajo una alícuota de 0.1 $\mathrm{ml}$ de la solución de $150 \mathrm{ml}$ donde fue enjuagada cada canal y se sembró en el medio selectivo, según lo descrito por Kuana et al. (2008), método recomendado por otros investigadores (Lindmark et al., 2009). Los ciegos fueron incididos longitudinalmente, la mucosa fue friccionada con un hisopo estéril y se frotó sobre el medio selectivo a modo de sembrado.

Se empleó el medio Campylobacter Blood-Free selective agar base (mCCDA, OXOID), suplementado con antibióticos (Campylobacter growth supplement, Oxoid), incubado a $42{ }^{\circ} \mathrm{C}$ por $48 \mathrm{~h}$, en atmósfera microaerofílica, usando un kit comercial (CampyGen ${ }^{\mathrm{TM}}$, OXOID) (Donnison, 2003; Kuana et al., 2008). Las colonias grisáceas, planas y que pueden tener brillo metálico son típicas de Campylobacter, considerándose como colonias positivas aquellas que además de ser Gram negativas, presentaban forma de espiral o de «S» al observarse en el microscopio. Las colonias de Campylobacter spp fueron tipificadas mediante pruebas bioquímicas de catalasa, oxidasa, hidrólisis de hipurato e hidrólisis del indoxil acetato, así como con pruebas de sensibilidad en placa al ácido nalidíxico y cefalotina (Donnison, 2003).

\section{Resultados y Discusión}

La frecuencia de Campylobacter spp fue de 16.7 y $26.7 \%$ en las canales y en los ciegos de pollos, respectivamente. Asimismo, el 60 y $73 \%$ de las muestras positivas en canales y ciego correspondieron a $C$. jejuni y el resto a $C$. coli (Cuadro 1).

Actualmente en el Perú, 400 millones de aves son producidas y consumidas al año $y$, aunque no existen reportes oficiales al respecto, los expertos calculan que el $75 \%$ de la producción se beneficia en centros de matanza no autorizados o clandestinos y en forma artesanal en los mercados del país (Málaga, 2011).

La presencia de Campylobacter en muestras de carnes dentro de las países de la Comunidad Europea es muy variada presentándose valores altos (>20\%) en seis países (EFSA, 2010). Suzuki y Yamamoto (2009) indican que la prevalencia de Campylobacter spp en carne de pollo es usualmente elevada 
Cuadro 1. Frecuencia de muestras de canales y vísceras intestinales de pollo a Campylobacter spp obtenidas de tres centros de acopio de aves en Lima Metropolitana (2009)

\begin{tabular}{cccccc}
\hline \multirow{2}{*}{ Muestras } & Total de & \multicolumn{2}{c}{$\begin{array}{c}\text { Positivas a } \\
\text { Campylobacter }\end{array}$} & $\begin{array}{c}\text { Positivas a } \\
\text { C. jejuni } \\
\text { muestras }\end{array}$ & $\begin{array}{c}\text { Positivas a } \\
\text { C. coli } \\
\text { (n) }\end{array}$ \\
\cline { 2 - 6 } & & $\mathrm{n}$ & $\%$ & & \\
\hline Canales & 60 & 10 & 16.7 & 6 & 4 \\
Ciego & 30 & 8 & 26.7 & 6 & 2 \\
\hline
\end{tabular}

$(>50 \%)$, a diferencia de otras carnes y otros alimentos de consumo común, donde las frecuencias son mínimas (EFSA, 2010).

La frecuencia de Campylobacter ssp obtenida en canales fue similar a reportes de $15 \%$ en centros de beneficio en Dinamarca, $y$ al $13-14 \%$ en carne de pollos de engorde vendidos en mercados de España y Holanda, respectivamente (EFSA, 2010). Sin embargo, existen diversos reportes con tasas mayores (Suzuki y Yamamoto, 2009; EFSA, 2010), teniéndose numerosos factores que afectan la prevalencia de Campylobacter. Asimismo, la uniformidad de lote es un parámetro que refleja el buen estado de salud a nivel de granja, y es un factor de protección contra contaminación de la carne con Campylobacter (Malher et al., 2011). La mejora de las prácticas de faenamiento es otra forma de mitigar Campylobacter en las canales; no obstante, en centros de beneficio con sistemas HACCP implementados se han detectado prevalencias elevadas de este patógeno (EFSA, 2010).

Se ha demostrado que las canales de aves beneficiadas al inicio del día, resultan protegidas de contaminación cruzada con Campylobacter desde otros lotes. Además, la cadena de procesamiento se presenta limpia y desinfectada antes de iniciar el beneficio del día. La matanza realizada a temperatura ambiental con un máximo de $15{ }^{\circ} \mathrm{C}$ (du- rante los meses fríos) se relaciona con un menor porcentaje de prevalencia de Campylobacter en la carne (Hue et al., 2010); asimismo, la edad del ave, el tipo de ave (mayor prevalencia en pollos orgánicos o criadas extensivamente), el método analítico, y el centro de beneficio (en Europa varían entre 4.9 a $100 \%$ ), afectan las tasas de prevalencias (EFSA, 2010; Hue et al., 2010). En el presente trabajo, las muestras fueron tomadas en los primeros minutos de iniciada la matanza y en horas de madrugada, donde la sensación térmica fue baja, y donde las aves que llegaban al centro presentaban un aparente buen estado de salud (lotes uniformes).

Existe una presencia mayor del $C$. jejuni en los alimentos con respecto a las demás especies del género, lo cual concuerda con los resultados del presente estudio; sin embargo, $C$. coli ha sido más frecuentemente aislado en Sudamérica, lo que hace posible que exista una vinculación del medio ambiente y el consumo de alimentos con la mayor frecuencia de aislamiento de $C$. coli como agente de diarrea en esta región (Fernández, 2011).

La tasa de aislamiento encontrado en el hisopado de ciego representa también un riesgo potencial de contaminación para los consumidores. El EFSA (2010) establece que la frecuencia de Campylobacter en las heces está entre un 20-25\%, aunque se han repor- 
tado en niveles superiores (Di Ginnatale et $a l .$, 2010). En el Perú, Tresierra-Ayala et al. (1996), observaron frecuencias de aislamientos de Campylobacter spp de $37 \%$ en heces de aves criadas en Iquitos.

Se ha demostrado que el porcentaje de canales positivas a Campylobacter están en relación a la cantidad de positivos a nivel de granja. La presencia del agente en el contenido intestinal está relacionada a una serie de factores, principalmente los vinculados a las prácticas de crianza. Además, se sabe que la contaminación aumenta con la edad del ave (Hue et al., 2010). Una disminución del microorganismo en las heces reduciría enormemente la presentación de la zoonosis en el hombre (Malher et al., 2011).

La campylobacteriosis como problema de salud pública en los países en desarrollo se presenta principalmente por alimentos mal procesados, pobremente preparados o preparados con deficiencias en higiene y sanidad. Las ETA se presentan con elevada frecuencia, afectando especialmente a los sectores de bajos recursos y a los grupos más susceptibles (ancianos, niños e inmunosuprimidos), en parte debido a la falta de conocimientos técnicos básicos sobre las buenas prácticas de manufactura (FAO, 2009). Es así que el Campylobacter en estos países es un frecuente agente etiológico de diarrea en niños menores de dos años (Black et al., 1989).

Puesto que la dosis infectiva de Campylobacter es pequeña, la limpieza común de las superficies con agua y detergente no elimina el riesgo de contaminación cruzada en la cocina (Cogan et al., 1999). Estudios indican que muestras positivas a Campylobacter en superficies de mesas, tablas de picar, platos y manos son transferidas desde la carne cruda de pollo durante su preparación (de Boer y Hahné, 1990). También se ha demostrado la contaminación cruzada desde carne cruda de pollo hacia tablas de picar y desde estas hacia vegetales de consumo crudo como lechugas y pepinos, a pe- sar del uso de desinfectantes para limpiar las tablas de picado posterior al cortado de carne (Zhao et al., 1998). Resultados similares obtuvieron Cogan et al. (1999) cuando muestrearon tablas de picar, superficies de trabajo, manos, toallas de manos, manteles, cuchillos, condimentos y sitios limpiados con el procedimiento de limpieza cotidiano, indicando que el uso de detergente, agua caliente e hipoclorito, si bien no elimina totalmente el Campylobacter, produce una reducción significativa del agente.

En el Perú no se conoce la real dimensión de la Campilobacteriosis humana debido a la no confirmación de casos y al subregistro de casos. Sin embargo, se dispone de un estudio que reporta $13 \%$ en niños menores de 2 años y $20 \%$ en niños menores de 6 meses, supuestamente protegidos con el solo consumo de leche materna (Perales et al., 2002), reflejando las condiciones precarias de higiene del grupo afectado.

En el país la carne de pollo difícilmente se come cuando está poco cocida, por lo que la contaminación cruzada en el hogar sería la principal vía de transmisión, especialmente si se toma en cuenta que sobrevive a los procedimientos de limpieza caseros. Se ha determinado que las cepas aisladas de pacientes infectados con $C$. jejuni guardan una relación genética significativa con cepas aisladas de carne fresca de pollos de engorde (Lindmark et al., 2009) y, además, que las cepas detectadas en la carne de pollo son las de mayor virulencia para el humano (Tsai et al., 2006; Pope et al., 2007), lo que permite señalar que la presencia de Campylobacter en la carne de mayor consumo en Lima evidencia el potencial problema de salud pública al confirmar el principal reservorio.

\section{Conclusiones}

La frecuencia de aislamiento de Campylobacter spp en canales y ciegos de pollos procedentes de centros de acopio de 
la ciudad de Lima fue de 16.7 y $26.7 \%$, respectivamente, siendo en su mayoría cepas de C. jejuni.

\section{Literatura Citada}

1. Adedayo O, Kirkpatrick B. 2008. Campylobacter jejuni infections: update on presentation, diagnosis, and management. Hosp Physician 44: 9-15.

2. Black RE, López de Romaña G, Brown KH, Bravo N, Grados O, Creed $H$. 1989. Incidence and etiology of infantile diarrhea and major routes of transmission in Huascar - Perú. Am J Epidemiol 129: 785-798.

3. Cogan TA, Bloomfield SF, Humphrey TJ. 1999. The effectiveness of hygiene procedures for the prevention of crosscontamination from chicken carcasses in the domestic kitchen. Lett Appl Microbiol 29:354-358.

4. Daniel W. 1996. Bioestadística: Base para el análisis de las ciencias de la salud. $3^{\mathrm{a}}$ ed. México: Limusa. 878 p.

5. de Boer E, Hahné M. 1990. Crosscontamination with Campylobacter jejuni and Salmonella spp. from raw chicken products during food preparation. J Food Prot 53: 1067-1068.

6. Di Ginnatale E, Prencipe V, Colangeli P, Alessiani A, Barco L, Staffolani M, Tagliabue S, et al. 2010. Prevalence of thermotolerant Campylobacter in broiler flocks and broiler carcasses in Italy. Vet Italiana 46: 415-423.

7. Donnison A. 2003. Isolation of thermotolerant Campylobacter Review \& methods for New Zealand laboratories. New Zealand: Ministry of Health of New Zealand, Enteric Zoonotic Disease Research in New Zealand Steering Committee. $79 \mathrm{p}$.

8. [EFSA] European Food Safety Authority. 2010. The community summary report on trends and sources of zoonoses, zoonotic agents and foodborne outbreaks in the European Union in 2008. EFSA J 8(1): 1496.
9. [FAO] Organización de las Naciones Unidas para la Agricultura y la Alimentación. 2009. Enfermedades transmitidas por alimentos y su impacto socioeconómico. Informe técnico sobre ingeniería agrícola y alimentaria. Roma. 194 p. [Internet]. Disponible en: ftp:// ftp.fao.org/docrep/fao/011/i0480s/ i0480s.pdf

10. Fernández H. 2011. Campylobacter y campylobacteriosis: una mirada desde América del Sur. Rev Peru Med Exp Salud Públ 28(1): 121-27.

11. Friedman CR, Hoekstra RM, Samuel $M$, Marcus R, Bender J, Shiferaw B, Reddy $S$, et al. 2004. Risk factors for sporadic Campylobacter infection in the United States: a case-control study in FoodNet sites. Clin Infect Dis 38 (Suppl 3): 285-296.

12. Hue O, Le BS, Laisney MJ, Allain V, Lalande F, Petetin I, Rouxel S, et al. 2010. Prevalence of and risk factors for Campylobacter spp contamination of broiler chicken carcasses at the slaughterhouse. Food Microbiol 27: 992-999.

13. Humphrey T, O'Brien S, Madsen M. 2007. Campylobacters as zoonotic pathogens: a food production perspective. Int J Food Microbiol 117: 237-257.

14. Kuana SL, Santos LR, Rodrigues LB, Borsoi A, Kellermann A, Salle CTP, et al. 2008. Pré-enriquecimento e isolamento direto para identificação de Campylobacter em swabs cloacais e carcaças de frango. Acta Scie Vet 36(1): 21-24.

15. Lindmark H, Boqvist S, Ljungström M, Ågren P, Björkholm B, Engstrand L. 2009. Risk Factors for Campylobacteriosis: an epidemiological surveillance study of patients and retail poultry. J Clin Microbiol 47: 2616-2619.

16. Málaga A. 2011. Plantas de beneficio peruanas: Hora Cero. Actualidad Avipecuaria. [Internet], [6 enero 2011]. Disponible en: http://www.actualidadavipecuaria.com/articulos/plantasde-beneficio-peruanas-hora-cero.html 
17. Malher X, Simon M, Charnay V, Danguy des Déserts R, Lehébel A, Belloc C. 2011. Factors associated with carcass contamination by Campylobacter at slaughterhouse in cecal-carrier broilers. Int J Food Microbiol 150: 8-13.

18. [MINAG] Ministerio de Agricultura. Industria Avícola: Junio 2010 [Internet], [04 agosto 2010]. Disponible en: www.minag.gob.pe

19. Murga H, Huicho L, Guevara G. 1993. Acute diarrhoea and campylobacter in Peruvian children: a clinical and epidemiologic approach. J Trop Pediatr 39: 338-341.

20. Oberhelman RA, Gilman RH, Sheen P, Cordova J, Taylor DN, Zimic M, et al. 2003. Campylobacter transmission in a Peruvian shantytown: a longitudinal study using strain typing of Campylobacter isolates from chickens and humans in household clusters. J Infect Dis 187:260-269.

21. Park R, Griffiths PL, Moreno GS. 1991. Sources and survival of Campylobacters: relevance to enteritis and the food industry. Soc Appl Bacteriol Symp Ser 20: 97S-106S.

22. Perales M, Camiña M, Quiñones $C$. 2002. Infección por Campylobacter y Shigella como causa de diarrea aguda acuosa en niños menores de dos años en el distrito de La Victoria, Lima - Perú. Rev Peru Med Exp Salud Púb 19(4): 186-192.

23. Pope C, Wilson J, Taboada E, MacKinnon J, Alves, Nash J, et al. 2007. Epidemiology, relative invasive ability, molecular characterization, and competitive performance of Campylobacter jejuni strains in the chicken gut. Appl Environ Microbiol 73): 7959-7966.

24. Robinson DA. 1981. Infective dose of Campylobacter jejuni in milk. Br Med J 282: 1584 .

25. Suzuki H, Yamamoto S. 2009. Campylobacter contamination in retail poultry meats and by-products in the world: a literature survey. J Vet Med Sci 71:255-261.

26. Tresierra-Ayala, Bendayan $M$, Bernuy A, Pereyra G, Espinoza $F$. 1996. Campylobacters termotolerantes en aves de la ciudad de Iquitos. Folia Amazónica 7: 187-194.

27. Tsai HJ, Huang HC, Tsai HL, Chang CC. 2006. PCR-based restriction fragment length polymorphism (RFLP) analysis of Campylobacter jejuni isolates from humans, chickens and dogs in northern Taiwan. J Vet Med Sci 68: 815-819.

28. Vellinga A, Van Loock F. 2002. The dioxin crisis as experiment to determine poultry-related Campylobacter enteritis. Emerg Infect Dis 8: 19-22.

29. [WHO] World Health Organization. 2000. The increasing incidence of human Campylobacteriosis. Report and Proceedings of a WHO Consultation of Experts. Copenhagen: WHO.

30. Zhao P, Zhao T, Doyle MP, Rubino JR, Meng J. 1998. Development of a model for evaluation of microbial crosscontamination in the domestic kitchen. $\mathrm{J}$ Food Prot 61: 960-963. 